\title{
A Classic Representation of Rare Susac Syndrome in young Pregnant Female !!
}

\author{
Geetesh Manik$^{1}$, Rajendra Pandey², Chinky Sharma ${ }^{3}$ \\ ${ }^{1}$ Interventional Cardiologist, ${ }^{2}$ Consultant Neurologist, ${ }^{3}$ Department of Medicine; Teerthanker Mahaveer Medical College \& Research \\ Centre, Moradabad, Uttar Pradesh, India.
}

\section{Corresponding Author:}

Dr. Geetesh Manik

Email: geetesh.manik@gmail.com

This is an Open Access article distributed under the terms of the Creative Commons Attribution License (creativecommons.org/ licenses/by/3.0).

Received : December 8, 2017

Accepted : April 5, 2018

Published : May 20, 2018

\begin{abstract}
Background: Susac syndrome, a rare disease is characterized by a clinical triad of subacute encephalopathy, visual loss secondary to retinal branch occlusions and sensorineuronal hearing loss. Case Report: We hereby report a 25 years old pregnant (7 months amenorrhea) female who presented with loss of vision of the left eye, tinnitus of the right ear and mild headache. Fundus evaluation showed branched retinal arterial occlusions in the left eye. A mild-moderate intensity right sided sensorineural hearing loss was observed on audiometry. Magnetic resonance images showed a hyper-intense lesion in the body of corpus callosum and peri-ventricular region. Patient was well managed with high dose steroids. Conclusion: A high index of suspicion of Susac syndrome is important as timely administration of immunosuppressive treatment may prevent permanent neuropsychological or visual sequelae.
\end{abstract}

Keywords: Deafness, Headache, Hearing Loss, Retinal Artery Occlusion, Susac Syndrome, Tinnitus.

\section{Introduction}

Susac's syndrome (SS) first described in 1976 has been well reported in Western literature (304 cases) though only 3 cases had been reported yet in Indian literature [1]. SS is a rare disease with a putative autoimmune vasculitic mechanism resulting in micro-angiopathy affecting the arterioles of the brain, retina and cochlea. It comprises a classical clinical triad of encephalopathy, visual loss secondary to retinal branch occlusions and sensorineuronal hearing loss [2,3]. We hereby represent this rare syndrome in young pregnant female.

\section{Case Report}

A 25-year-old pregnant female with 7 months amenorrhea was referred to cardiology outpatient from obstetrics department to rule out any cardiac anomaly as patient presented with sudden onset loss of vision in left eye (embolic phenomenon).
On detailed evaluation patient reported loss of vision of the left eye since 9 days, decreased hearing with tinnitus of the right ear and mild headache. She was previously healthy and had no remarkable medical or ocular history. Patient was referred to ophthalmology and neurology for detailed evaluation. Color fundus photography of her left eye showed ischemic retinal edema inferio-temporally and "cherry red spot" suggestive of retinal artery branch occlusion [Fig.1]. On neurological evaluation there was no evidence of increased intracranial pressure (ICP). EEG (electroencephalogram) did not show any abnormality. Her blood investigation for vasculitis (full blood count, erythrocyte sedimentation rate, fasting lipids and glucose, autoantibody screen including anti-cardiolipin antibody, protein $\mathrm{S}$ and C levels, and anti-thrombin III level) work up was negative. She had no features of increased intracranial pressure (ICP). The T2-weighted MRI scan showed high-signal intensity around 
the peri-ventricular region and in the corpus callosum [Fig.2a, 2b]. Her cardiac evaluation was also within normal limit. An audiogram showed moderate sensorineural hearing loss in the right ear. She was treated with high dose of steroids (methyl-prednisolone $1 \mathrm{gm} /$ day) for 5 days, and then tapering dose of oral steroids at the time of discharge. She showed significant improvement in her headache with some recovery of left eye vision at the time of discharge.

\section{Discussion}

Susac syndrome was first described by Susac in 1976 [1]. Female predominance with a sex ratio of 3 to 1 is reported. The mean age of presentation is 28 years with a range of $9-58$ years [4]. It consists of a clinical triad of acute or subacute encephalopathy which could manifest in a broad spectrum of symptoms, e.g. memory impairment, confusion, behavioral disturbances, ataxia, dysarthria, paranoid psychosis, and headaches, sensorineural hearing loss at low and medium frequencies and branch retinal artery occlusions leading to scotomata and vision distortion. All clinical features may not be present at the same time, and may in fact fluctuate over a 1-2 year period, which often leads to delay in diagnosis [5].

The primary pathology is likely to be an autoimmune endotheliopathy (microangiopathy) affecting small vessels, with subsequent embolization, of the brain, retina, and inner ear. Autopsy studies done in past on patients with Susac syndrome suggested that the syndrome could be an endothelioapathy $[6,7]$. The syndrome is often misdiagnosed and differentials include multiple sclerosis, disseminated encephalomyelitis, lupus erythematosus, Ménière disease, migraine and thromboembolic strokes [7]. Interestingly, most of the patients do not present initially with the classical clinical triad and show incomplete forms of the disease either presenting with only branch retinal artery obstruction or hearing loss in the absence of encephalopathy. Hence, diagnosing Susac syndrome is always challenging [8].

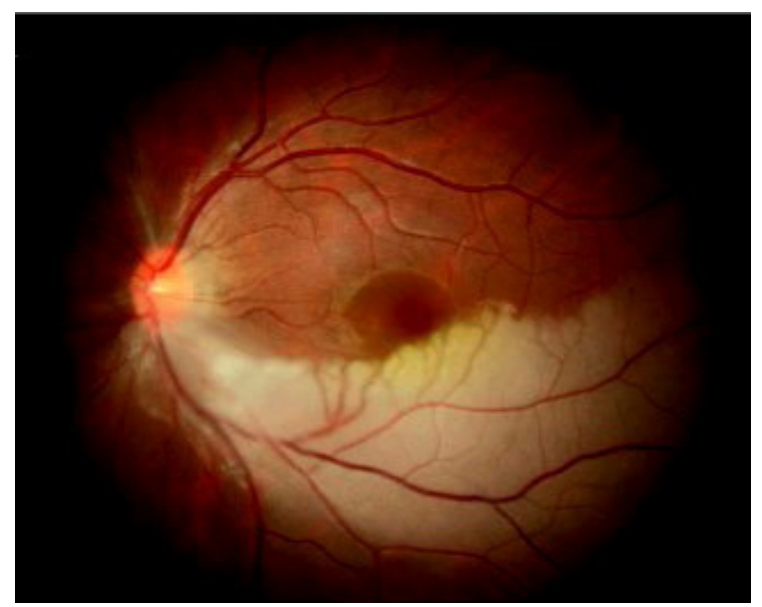

Fig.1: Fundus of left eye showing retinal edema inferiotemporally and cherry red spot.
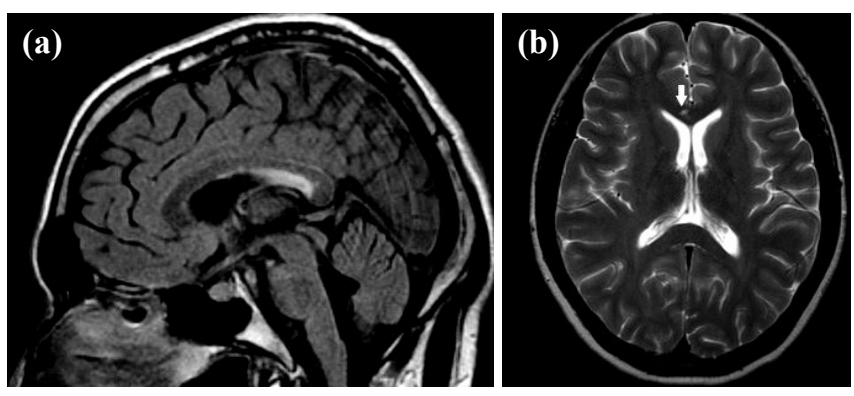

Fig.2(a): $M R I T 2 W$ showing hyper-intensity in body of corpus callosum. (b): MRI T2W showing hyper-intensity in peri-ventricular region.

Brain MRI, fluorescein angiography and audiometry are essential for correct diagnosis. Involvement of the corpus callosum, in particular, evidence of 'snowball lesions' is considered a characteristic sign of this syndrome. Many lesions in the corpus callosum become cystic within a period of 4 weeks, which is highly suggestive of micro-infarcts rather than demyelination, and it can be used to differentiate demyelinating disease. Multiple sclerosis can be differentiated based on these findings. Fluorescein angiography shows characteristic retinopathy with multiple retinal artery occlusions. The bilateral or unilateral hearing loss is due to cochlear involvement. Hearing loss may be asymptomatic and found only by audiometry. This hearing loss typically involves low and medium frequencies [9]. 
Main therapy for treatment of Susac syndrome is immunosuppressive like corticosteroids, immunosuppressive agents, anti-platelet agents, anticoagulant agents and plasmapheresis. Although their efficacy remains difficult to evaluate, early recognition of the disease is important because treatment with immunosuppression seems to reduce permanent cognitive, audiologic, and visual sequelae [10].

\section{Conclusion}

Susac syndrome is a rare disease characterized by an axiomatic triad of encephalopathy, visual loss secondary to retinal branch occlusions and sensori-neuronal hearing loss. A high index of suspicion leading to early recognition of this syndrome is important as timely administration of immunosuppressive treatment may prevent permanent neuropsychological or visual sequelae.

Contributors: GM treated the patient, collected the data, and wrote the manuscript. RP did neurological evaluation and manuscript editing. CS assisted in patient management and contributed in manuscript writing. GM will act as guarantor. All authors read and approved the final manuscript. Funding: None; Competing interests: None stated.

\section{References}

1. Eluvathingal Muttikkal TJ, Vattoth S, Keluth Chavan VN. Susac syndrome in a young child. Pediatr Radiol. 2007;37:710-713.

2. Kleffner I, Duning T, Lohmann H, Deppe M, Basel T, Promesberger J, et al. A brief review of Susac syndrome. J Neurol Sci. 2012;322:35-40.

3. Susac JO, Hardman JM, Selhorst JB. Microangiopathy of the brain and retina. Neurology. 1979;29:313-316.

4. Susac JO, Murtagh FR, Egan RA, Berger JR, Bakshi $\mathrm{R}$, Lincoff $\mathrm{N}$, et al. MRI findings in Susac's syndrome. Neurology. 2003;61:1783-1787.

5. Do TH, Fisch C, Evoy F. Susac syndrome: report of four cases and review of the literature. Am J Neuroradiol. 2004;25:382-388.

6. Susac JO, Egan RA, Rennebohm RM, Lubow M. Susac's syndrome: 1975-2005 microangiopathy/autoimmune endotheliopathy. J Neurol Sci. 2007;257:270-272.

7. Buzzard KA, Reddel SW, Yiannikas C, Sean Riminton D, Barnett MH, Hardy TA. Distinguishing Susac's syndrome from multiple sclerosis. J Neurol. 2015;262:1613-1621.

8. Saw VP, Canty PA, Green CM, Briggs RJ, Cremer PD, Harrisberg B, et al. Susac syndrome: microangiopathy of the retina, cochlea and brain. Clin Experiment Ophthalmol. 2000;28:373-381.

9. Saenz R, Quan AW, Magalhaes A, Kish K. MRI of Susac's syndrome. Am J Roentgenol. 2005;184:16881690.

10. Papo T, Biousse V, Lehoang P, Fardeau C, N'Guyen N, Huong DL, et al. Susac syndrome. Medicine (Baltimore) 1998;77:12-40. 